\title{
Location Analytics for Transitioning to Fire Resilient Landscapes
}

\author{
Alan T. Murray \\ Department of Geography \\ University of California at Santa \\ Barbara \\ amurray@ucsb.edu
}

\author{
Richard L. Church \\ Department of Geography \\ University of California at Santa \\ Barbara \\ rickchurch@ucsb.edu \\ Peter Stine \\ Pacific Southwest Research \\ Station, U.S. Department of \\ Agriculture Forest Service \\ Peter.Stine@usda.gov
}

\author{
B. Amelia Pludow \\ Department of Geography \\ University of California at Santa \\ Barbara \\ bapludow@ucsb.edu
}

\begin{abstract}
Wildfire risk is significant for forest and vegetative landscapes, particularly in regions where climate change is resulting in prolonged droughts and extended fire seasons that are a fire risk to people and property. An important component of mitigation is restoration programs that transition landscapes to be more fire resilient. A collaborative partnership between the US Forest Service and university researchers is reported that takes advantage of location intelligence. This paper reviews this general planning problem and details location analytic based approaches for informing mitigation efforts. Application of results highlight the ability to optimize goals and objectives while maintaining project area needs and treatment thresholds.
\end{abstract}

\section{Introduction}

Wildfire is an ever-present feature of forests and shrublands in the western United States characterized by vegetation that has historically been subject to fire as part of its regular lifecycle. Development, fire suppression, logging of the largest, most fire-resistant trees, and climate change have fundamentally altered natural regenerative processes, and as a result created a range of wildfire vulnerabilities for flora, fauna, humans, critical infrastructure, etc. Recent wildfires highlight the devastation faced. In northern California alone during 2020 there was the August Complex (burning over 1 million acres, destroying nearly 1,000 structures, one death and multiple injuries), the SCU Lightning Complex (burning approximately 400,000 acres and destroying over 200 structures), the Creek (burning nearly 380,000 acres, destroying over 60 structures and one fatality) and the LNU Lightning Complex (burning some 360,000 acres, destroying almost 1,500 structures and multiple fatalities and injuries) and the North Complex (burning nearly 320,000 acres, destroying and damaging some 2,500 structures and 15 fatalities), among others. Overall, wildfire covered more than 4 million acres, destroyed more than 10,000 structures, and killed 31 people in California in 2020. Further, 17 of the 20 largest wildfires in California history have happened in the last 20 years.

As large as these recent fires seem in total, researchers have estimated that prior to $1800,1.8$ million hectares (4.5 million acres) of grasslands, forests, and oak woodlands burned each year in California as a result of lightning and native American Indian practices [25]. Native Americans regularly burned forest understory by fire as an aid in hunting and gathering. It is estimated that the summer and fall of California were characterized by smoky air during the prehistoric period [25]. So, one must recognize that fire has always been a significant feature in California landscapes, but also throughout the western United States more generally.

Even without native Americans using fire to alter landscapes for their needs, fire was still a dominant feature in the western United States. However, European settlement led to different landscape practices, including intense forest harvesting and land clearing followed by active fire suppression. Clear cut harvesting 
and selective harvesting resulted in removal of the largest trees, those generally 200-500 years old that are most resilient to wildfire. Fire suppression also had major impacts on forest composition, with significant shifts in species composition. Landscapes of shadeintolerant pines and oaks that once were common transitioned to shade tolerant firs and cedars. These species thrived under the denser forest conditions of fire suppression. Larger, established trees tend to survive regularly occurring natural fires, but smaller trees and downed fuels do not. Altogether, the lack of regular fire in forests has led to more dense stands, sometimes as many as 700 trees per acre as compared to presettlement levels of 100 trees per acre. Dense stands of trees have been likened to having too many straws in the ground competing for limited soil moisture. This intense competition for moisture during periods of drought puts undue stress on trees and other vegetation, making them more susceptible to disease and insect infestations, like bark beetles, and additionally contribute to more intense fires [15]. Droughts are a common occurrence in the dry summer climates of the western United States. In addition, climate change and varying warming trends result in earlier spring runoff and lower summer/fall soil moisture, further contributing to tree stress and mortality.

With a buildup of fuels, including accumulation of needle cast and branches, downed trees, dense stands of smaller trees, standing dead trees, and ladder fuels (fuels that help propagate a fire from the ground into tree canopies), fires are more intense and destructive. When forest stands are thinned while retaining some of the largest trees, ladder fuels are removed, and downed debris is burned or mechanically removed, forest stands can survive and even thrive in the presence of wildfire. Thus, restoration programs for forested and other landscapes are clearly more essential than ever for making them resilient to wildfire and drought. The focus in this paper involves the spatial planning of silvicultural strategies, i.e. prescribed burning, harvesting, tree thinning, and mechanically removing ladder fuels and litter. The intent is to shift the structure and composition of landscapes towards conditions within the natural range of variation, thereby helping to increase resiliency to disturbances. In addition, restoration may help to protect vulnerable and endangered habitat, supporting species conservation and protection efforts by preventing severe, highly destructive wildfires. Strategic planning is critical for such restoration efforts in order for them to have the most effective influence in mitigating wildfire risks. It should be noted that some elements that can contribute to higher forest fire severity, like snags (e.g., standing dead trees that decompose naturally, upright or on the ground) and ladder fuels (e.g., broken and/or dead branches, leaves, vegetation, etc.), can also provide needed habitat elements, providing nesting cavities for birds and animals and a rich insect environment that can support woodpeckers and other bird species [11].

Restoration projects can be costly and require considerable oversight, making strategic investment and planning a necessity. An important part of the restoration process involves identification of project areas, or patches, comprised of smaller stands. This must be done in the context of achieving wildfire mitigation goals, but also practical limitations on total restoration treatments possible.

This paper details location intelligence approaches to support restoration planning efforts intended to reduce wildfire risks. The next section provides background review of wildfire risks and associated research in this area. This is followed by location analytic approaches that can be used in strategic planning efforts. Application results involving the Stanislaus National Forest are then reported. The paper ends with discussion and conclusions.

\section{Background}

Over the last 15 years, the US Forest Service has begun to devote more resources towards the protection of critical habitat and Wildland-Urban Interface lands from catastrophic fires, events that can significantly damage if not destroy habitats of species at risk such as the California spotted owl. The US Forest Service manages 18 National Forests in California that comprise about $81,000 \mathrm{sq} \mathrm{km} \mathrm{(31,000} \mathrm{sq.} \mathrm{mi.),} \mathrm{approximately} \mathrm{the}$ size of Austria. All but two of these National Forests have some form of fuels removal planning underway. These fuel reduction operations have been hampered by limited budgets, litigation by environmental organizations, and concerns regarding their effectiveness. Moritz et al. [19] note that thinning the density of smaller trees can decrease fire intensity, but if the slash (non-merchantable tree tops and limbs) are not removed this material will increase the surface fuels which is typically a significant contributor to fire intensity and spread. Treating the ladder and surface fuels is costly and thus limits overall acreage than can be managed within limited budgets. Keeping the largest trees in a forest stand aids in promoting a diversified structure and overall fire resilience, but at an additional cost as the largest of the trees are often the most merchantable. Consequently, comprehensive fuels removal can cost more than the value of the timber being harvested by thinning operations.

The good news is that not all stands of a forest need to be treated. Finney [13] demonstrated through the use of fire simulation models that when strategically treating about $25 \%$ of the landscape, fire behavior tended to act 
as if the whole forest had been treated. Collins et al. [9] also demonstrated that selective fuels treatments can reduce the speed and intensity of forest fires. Lydersen et al. [18] found that fuels treatments and controlled burns significantly reduced the speed and intensity of the 2013 Rim fire in the Stanislaus National Forest and Yosemite National Park. At more than 250,000 acres, this is one of the largest forest fires that has occurred in California. Since the work of Finney [13], the US Forest Service has been involved in the planning and management of fuels removal for selected areas across the National Forests in California [8].

To aid in planning fuels treatments, spatially-based models and software programs have been developed to identify critical areas for fuels removal as well as schedule these activities over time [8]. One of the models that has been used extensively in the western United States is the ForSys program (see [1,2]). The essence of the ForSys program is to select those areas that score the highest in value as computed by a composite multi-objective value function. The application of programs like ForSys may involve spatial units that are often either too large or too small to be considered feasible project areas. For example, ForSys has been applied to 30m-by-30m cells (or .09 ha) [2]. If the desired project size is approximately 40 ha (100 acres) in order to be economically and operationally feasible for a logging company, then selected cells and/or management units need to be aggregated into groups of approximately 450 cells. What complicates the grouping of selected cells into projects is that the patch to be treated should be contiguous to the greatest extent possible while still meeting management objectives such as minimizing travel time, avoiding movement across ecologically sensitive areas like streams, and treating areas that are most in need. If a project patch is not contiguous, then the cost of treatment increases substantially as equipment and operations must be moved between any discontinuities that exist within a project area. Unfortunately, creating a contiguous patch from spatial units (like the $30 \mathrm{~m}-$ by$30 \mathrm{~m}$ cells) means that some units may need to be selected to meet the spatial contiguity requirements that are not necessarily the most beneficial individual units. This is the main problem addressed in this paper.

The literature on grouping spatial units into contiguous areas as a spatial optimization problem began with Garfinkel and Nemhauser [14], where counties in Washington were aggregated into contiguous political districts. Spatial districting models have subsequently been developed to support a wide variety of planning applications, including transportation and economic forecasting [16], economic comparison regions [12], and racially balanced school districts [22].
Whereas districting and $p$-regions problems require the grouping or selection of all spatial units into contiguous groups, the problem faced by forest planners is not grouping all land management units into project areas, but grouping only a subset of areas into feasible projects or patches. Nevertheless, all of the districting, regions delineation and patch models are quite difficult to solve optimally because of the inherent contiguity requirements. There are a number of notable mathematical structures that have been proposed to enforce spatial contiguity while finding one or a set of optimal patches, defined in terms of contiguous spatial units that meet specific constraints and optimize an objective (see [12,27]). These include flow-based contiguity constraints [23], order-based contiguity conditions [10], tree-based structures [12], densitybased constraints and objectives [17] and path-based contiguity constraints [5]. In addition, heuristics have been developed for these types of spatial optimization problems $[2,6]$.

In the next section we give a more detailed description of the patch design model developed for the US Forest Service along with an initial application to a region of the Stanislaus National Forest in California.

\section{Location analytics}

The importance and significance of location intelligence has long been recognized. Indeed, the phrase "location, location, location" emphasizes just how critical aspects of spatial interactions, travel patterns, proximity, access, accessibility, etc. are to the success (or failure) of locational choices for a business, service provider and/or planned activity. Accordingly, analytics and GIS have played a critical role in codifying, quantifying, and measuring, geographic environments, enabling knowledge to be obtained through extraction, summary, and computation. Spatial information and simple summary measures / statistics are most certainly one aspect of location intelligence. Advanced analytics build on this by enhancing capabilities tremendously in a number of important ways.

Location intelligence can be obtained in any number of forms. It is helpful to differentiate between descriptive, predictive and prescriptive approaches, each offering insight and knowledge in different ways. As an example, GIS is particularly effective at describing a geographic environment through the representation of information in different layers, each reflecting objects of some particular type along with associated attributes. Statistical methods like spatial regression, as another example, offer capabilities to both describe relationships and predict likely attribute values given observed characteristics. Prescriptive approaches 
inform planning, management and policy by indicating where and to what degree something should happen. As an example, if one seeks the safest evacuation route during a wildfire, then a prescriptive approach will identify a travel path under the stipulated conditions.

The remainder of this section focuses on prescriptive aspects of location intelligence through the use and application of spatial optimization approaches. Reviews and discussion of spatial optimization can be found in Church [4] and Tong and Murray [26]. The essence of spatial optimization is the use of decision variables to reflect prescriptive actions of what should be done in order to achieve a desirable outcome. Spatial characteristics may be present in terms of geographic decisions regarding location, allocation and/or routing, objectives and/or constraints that are geographic in nature, or through attributes, coefficients and sets that are derived based on geographic conditions.

The use of location analytics to support a range of prescriptive contexts is detailed in Murray [20]. Of particular significance for wildfire mitigation efforts is the knapsack problem summarized in Church and Murray [7]. The formulation of the knapsack problem is the following:

$$
\begin{array}{cc}
\text { Maximize } & \sum_{i} \beta_{i} X_{i} \\
\text { Subject to } & \sum_{i}^{i} \alpha_{i} X_{i} \leq T \\
& X_{i}=\{0,1\} \quad \forall i
\end{array}
$$

where $i$ is the index of land management units, $\beta_{i}$ is the benefit of treating unit $i, \alpha_{i}$ is the area of unit $i, T$ is the threshold on a project area and $X_{i}$ is the decision variable indicating treatment $\left(X_{i}=1\right)$ or no treatment $\left(X_{i}=0\right)$ for unit $i$. The interpretation of benefit, $\beta_{i}$, is clearly subjective, reflecting the intent of the modeling process. In this case, $\beta_{i}$ is utilized to reflect the value of treatment of a given land management unit $i$. More discussion of this model input / parameter if offered in the application section below.

The knapsack approach has been extensively relied upon to address wildfire risk mitigation by the US Forest Service (see [1]) as well as by international agencies (see [3]). The intent of the model, (1)-(3), is to identify the best land units to treat in order to reduce the threat of wildfire, but doing so within the constraint (or budget) of total operations. That is, there is a limit on total activity possible due to personnel and budgets, so the idea is to identify the best treatment strategy possible within the constraining conditions. An interesting observation regarding the knapsack approach is that land unit selection is wholly based on the benefit in combination with impact on the threshold, agnostic of proximity to other selected land units. This may be problematic in many cases because treatments are carried out within the various limitations of on-theground operability, involving costs to position personnel and equipment as well as costs to move material to a processing facility. Accordingly, Ager et al. [1], Alcasena et al. [3] and others note the importance of identifying land units for treatment that form workable project areas, with such areas being contiguous in order to address treatment priorities and operability considerations.

A critical extension of the knapsack approach has been addressing issues of spatial contiguity. The definition of spatial contiguity is that any pair of selected units can be reached by travel through only adjacent/neighboring units that are also selected. Church et al. [6] detailed the need for connected and contiguous area in the selection of land for the conservation habitat critical to the kit fox. Shirabe [23] formalized a spatial optimization model for land unit selection that imposed contiguity between units, essentially extending the (1)-(3). The resulting model is the following:

$$
\begin{gathered}
\sum_{i} \beta_{i} X_{i} \\
\text { Maximize } \\
\sum_{i}^{i} \alpha_{i} X_{i} \leq T \\
\sum_{j \in N_{i}} Y_{i j}-\sum_{j \in N_{i}} Y_{j i} \geq X_{i} \\
\sum_{i} V_{i}=1 \\
\sum_{j \in N_{i}} Y_{i j} \leq(M-1) X_{i} \quad \forall i \\
X_{i}=\{0,1\} \forall i \\
V_{i}=\{0,1\} \forall i \\
Y_{i j} \geq 0 \forall i, j
\end{gathered}
$$

where notation remains the same as previously defined, with the following additions associated with contiguity: $N_{i}$ the spatial neighbors of unit $i$ (often defined based on adjacency), $M$ a large number, $V_{i}$ is the decision variable associated with whether or not unit $i$ is a sink and $Y_{i j}$ a decision variable indicating flow between units $i$ and $j$. What can be observed is that objective (4) is exactly the same as (1) in the knapsack problem, as is the threshold constraint (5) compared to (2). What is new and unique are the set of decision variables and constraints represented in (6)-(8), (10) and (11), which track contiguity as a network flow process to ensure that any selected land unit is connected to neighboring selected units. 
It is worth noting that temporal extension of these models is also possible, if treatment activity occurs over longer periods of time and there are impacts on local and regional benefits. In the present context, allocation of treatment activity is over a sufficiently short period of time, making the single period formulations most appropriate for the encountered decision making process.

A second point to note is that there has long been interest in contiguity in land use management, with Wright et al. [27] being an early attempt to indirectly model notions of contiguity. Specific to wildfire treatment planning efforts, recent work by Ager et al. [1], Alcasena et al. [3] and Pais et al. [21] all consider contiguity in land unit selection.

\section{Application results}

The Stanislaus National Forest consists of nearly 900,000 acres in the Sierra Nevada mountains of California. It is adjacent to Yosemite National Park and is easily accessible from the San Francisco Bay area, making it a popular recreation destination. The large majority of the forest landscape is shaped by fire; most recently, the 2013 Rim Fire was ignited within the Stanislaus, eventually spreading beyond its borders, and ultimately burned over 250,000 acres, as noted previously.

The unique setting of the forest, and the impact of the Rim Fire, led to the initiation of the Social and Ecological Resilience Across the Landscape (SERAL) project in the Stanislaus National Forest (Figure 1). This area consists of 117,000 acres within the Stanislaus, and the SERAL goal is to increase forest resilience from a socio-ecological perspective and move forest conditions within the natural range of variation [24]. SERAL consists of 17 Potential Operational Delineations, areas for which risk assessments are carried out and difficulty of suppression is analyzed. Additionally, a Potential Operational Delineations is expected to be the level at which wildfire control is organized.

Within the SERAL Potential Operational Delineations, the smallest unit of analysis is the land management unit. There are approximately 10,000 land management units in the SERAL region. They are delineated to be relatively homogenous in terms vegetation type and topography. Sizes of each unit range from 0.22 to 217.06 acres, with an average size of 11 acres. Using various methods of location prioritization, treatment is determined at the land management unit level, with a unit either treated or not treated. Partial unit treatments are possible, however, for prioritization analyses these are the units of selection using appropriate decision support tools such as ForSys.
For the purposes of this project, we have focused on the single Potential Operational Delineation shown in Figure 1, consisting of 582 land management units across 7,557 acres. The project threshold $(T)$ is set at 100 acres - a typical project size for this forest type. The benefit of treating a unit $\left(\beta_{i}\right)$ is determined by a US Forest Service derived resilience metric which integrates three primary forest structure variables measured with remote sensing instruments: mean clump size, trees per acre, and proportion of gaps. This forest structure metric is then compared to reference conditions to calculate a "departure" value for each land management unit. A higher value indicates conditions which are further from reference conditions, and expected to benefit more from treatment. For individual land management units, the resilience value ranges from 0 (no benefit from treatment) to 6.49 . Effectively the metric is structured to reflect the relative benefit of treating a land management unit compared to other units. Given linear objective functions, (1) and (4), an individual land management unit has a given total benefit, if treated. The total potential benefit possible if all units were treated is 750.71 , but this would clearly violate the threshold of 100 acres.

A desktop personal computer (Intel Xeon E5 CPU, $2.30 \mathrm{GHz}$ with $96 \mathrm{~GB}$ RAM) running Windows was used in this analysis. Data management, manipulations, analysis, and display were carried out using ArcGIS (version 10.8.1). A commercial optimization solver, Xpress (version 5.0.2), was used to solve each problem instance.

The plan identified using the knapsack approach, (1)-(3), suggests the selection of 36 units with a total benefit of 87.52 . Solution time is trivial in this case, requiring less than 1 second. The spatial arrangement of this planning configuration is shown in Figure 2. Given the intention of maximizing total benefit, (1), the selection of land management units may or may not form a contiguous project area. Indeed, Figure 2 demonstrates that spatial contiguity does not occur in this case, with few instances of neighboring units being selected for treatment. Beyond this, the dispersed nature of identified units means this solution is virtually impossible to implement due to project oversight and operational cost considerations.

Since the knapsack approach stipulated in (1)-(3) lacks an ability to impose or encourage contiguity, fragmented treatment units are likely. The result is that this is not a viable project from a management and implementation perspective. Thus, the extension to impose spatial contiguity, (4)-(11), is absolutely essential. Application of this spatial contiguity extension, where neighbors are defined as sharing a common polygon edge or point, results in 23 units being selected (still 100 acres total), giving a total benefit of 
46.95. The spatial arrangement of this treatment patch configuration is shown in Figure 3. Noteworthy in Figure 3 is that project operation now is confined to a more manageable local area. Treatment equipment and personnel can readily move between units, eliminating the need for more costly transportation and set-up services. Indeed, this is the rationale for imposing patch thresholds and contiguity requirements in the first place.

\section{Discussion}

There are a number of rather important discussion points regarding the treatment of landscapes in order to make them as resilient to wildfire as possible. Many issues revolve around operational concerns. In what follows we touch on the tradeoffs of requiring spatial contiguity, computational effort and potential extensions that relax rigid interpretations of contiguity.

As noted previously, the knapsack approach without contiguity requirements identified a treatment plan with a total benefit of 87.52 (Figure 2), while imposing only the threshold limit of 100 acres. The addition of a spatial contiguity requirement resulted in the ability to only achieve 46.95 total benefit (Figure 3 ). This is only $53.56 \%$ of the maximum that was not contiguous. Thus, there is a sacrifice in the total benefit to wildfire resiliency across the entire study area by requiring that a project be contiguous. This tradeoff between total benefit and an ability to provide for operational efficiency is inherent to the problem and such a sacrifice of benefit is necessary in practice.

A second issue to discuss involves the mechanics in solving the model. The use of a commercial solution package is convenient and facilitates operational implementation, but computational effort may well be a concern for solving any optimization model. This is indeed the case for the knapsack approach with spatial contiguity requirements, (4)-(11). The time required to solve the spatial contiguity extension is not trivial, requiring nearly 94 hours to verify optimality within $5 \%$. However, it should be noted that this best feasible solution was identified after 116 seconds, with the remainder of the time spent attempting to find a better solution and/or verifying that the solution identified is the best solution. Why is this the case? Model structure dictates whether an exact approach can be successful. This if generally a function of the number of decision variables combined with constraint structure. For integer programs, a typical approach involves linear programming relaxation followed by branch and bound to resolve decision variables that are required to be integer. When constraint structure produces fractional solutions in the linear program relaxation, this creates a need for branching and bounding. This is precisely what is encountered in this situation. Significant computational effort is devoted to identification and verification of an optimal integer solution, and this is only possible through the branch and bound process.

Of course, this paper has focused on only one patch or project area, so extension to multiple patches, a practical necessity for landscape level treatment strategies, can be expected to be even more challenging to solve. While not a focus in this paper, improved and/or alternative solution approaches are clearly very appealing, assuming they can find optimal or highquality treatment plans. A common alternative is a heuristic. Church [4] and Tong and Murray [26] discuss heuristics for spatial optimization problems, emphasizing that a heuristic is an approach applying steps and rules to find a solution, often ensuring that the solution is feasible, with the hopes that it is also of high quality. However a heuristic solution cannot be formally quantified or established, leaving uncertainty about quality of a solution that has been identified.

A final discussion point has to do with the imposition of spatial contiguity. As noted previously, contiguity is a property that has to do with units being mutually interconnected. Namely, a contiguous set of land management units is such that one could travel from a selected unit to any other selected unit through other selected units. Thus, a set of selected units is either contiguous or not contagious. An interesting notion introduced in Wu and Murray [28] is relative contiguity. They proposed a measurement and modeling approach for optimizing the selection of management units with respect to objective quality as well as relative contiguity. That is, they recognized that formal contiguity may not always be necessary and that some configurations of selected units are more contiguous than others. This is particularly relevant in the case of forest treatment planning for wildfire resiliency, where spatial units that comprise a project / patch may not always need to be contiguous. As a result, entertaining alternative approaches to strict contiguity might be a valuable avenue to explore as well as considering less strict definitions of adjacency using existing approaches discussed in this paper.

\section{Conclusions}

The primary thesis of this paper is that wildfire risk is significant for forest and vegetative landscapes, particularly in regions where climate change is increasing risk due to drought, the build-up of surface and ladder fuels, disease, etc. A critical management need in forest across most of the western United States is restoration programs that transition landscapes to be more fire resilient. Such a transition must be done in a manner that is efficient, effective, and affordable so that large portions of landscapes can become resilient as 
quickly as possible. Accordingly, planning for treatments to transition to fire resilient landscapes requires the support of locational intelligence. The spatial contiguity model, (4)-(11), is an important location intelligence aid, representing an analytic approach that addresses both threshold limits as well as interconnectivity between units to be treated. These are critical operational considerations, facilitating effective implementation and minimal costs. The reported findings for project identification on the Stanislaus National Forest demonstrate that viable treatment patches require the support of location analytics. Further, this is and remains a challenging task, with many computational considerations to be addressed. Extensions that account for multiple projects are critical as are the development of rigorous heuristics. Finally, it may be important to consider relaxations of strict contiguity interpretations, such as the notion of relative contiguity.

\section{Acknowledgements}

Funding for this research is through the Pacific Southwest Research Station of the U.S. Forest Service, Department of Agriculture.

\section{References}

[1] Ager, A. A., Day, M. A., \& Vogler, K. (2016). Production possibility frontiers and socioecological tradeoffs for restoration of fire adapted forests. Journal of Environmental Management, 176, 157-168.

[2] Ager, A. A., Houtman, R. M., Day, M. A., Ringo, C., \& Palaiologou, P. (2019). Tradeoffs between US national forest harvest targets and fuel management to reduce wildfire transmission to the wildland urban interface. Forest ecology and management, 434, 99-109.

[3] Alcasena, F. J., Ager, A. A., Salis, M., Day, M. A., \& Vega-Garcia, C. (2018). Optimizing prescribed fire allocation for managing fire risk in central Catalonia. Science of the total environment, 621, 872-885.

[4] Church, R. L. (2001). Spatial optimization models. In International Encyclopedia of the Social and Behavioral Sciences, edited by L. Smelser and P. Baltes, 1481114818. Elsevier.

[5] Church, R. L., \& Cova, T. J. (2000). Mapping evacuation risk on transportation networks using a spatial optimization model. Transportation Research Part C: Emerging Technologies, 8(1-6), 321-336.

[6] Church, R. L., Gerrard, R. A., Gilpin, M., \& Stine, P. (2003). Constructing cell-based habitat patches useful in conservation planning. Annals of the Association of American Geographers, 93(4), 814-827.

[7] Church, R. L., \& Murray, A. T. (2009). Business site selection, location analysis, and GIS. Hoboken, NJ: John Wiley \& Sons.
[8] Church, R. L., Niblett, M. R., O’Hanley, J., Middleton, R., \& Barber, K. (2015). Saving the forest by reducing fire severity: selective fuels treatment location and scheduling. In Applications of location analysis (pp. 173190). Springer, Cham.

[9] Collins, B. M., Stephens, S. L., Moghaddas, J. J., \& Battles, J. (2010). Challenges and approaches in planning fuel treatments across fire-excluded forested landscapes. Journal of Forestry, 108(1), 24-31.

[10] Cova, T. J., \& Church, R. L. (2000). Contiguity constraints for single-region site search problems. Geographical Analysis, 32(4), 306-329.

[11] DellaSala, D. A., \& Hanson, C. T. (2015). The ecological importance of mixed-severity fires: nature's phoenix. Elsevier.

[12] Duque, J. C., Church, R. L., \& Middleton, R. S. (2011). The p-Regions Problem. Geographical Analysis, 43(1), 104-126.

[13] Finney, M. A. (2001). Design of regular landscape fuel treatment patterns for modifying fire growth and behavior. Forest Science, 47(2), 219-228.

[14] Garfinkel, R. S., \& Nemhauser, G. L. (1970). Optimal political districting by implicit enumeration techniques. Management Science, 16(8), B-495.

[15] Jenkins, M. J., Page, W. G., Hebertson, E. G., \& Alexander, M. E. (2012). Fuels and fire behavior dynamics in bark beetle-attacked forests in Western North America and implications for fire management. Forest Ecology and Management, 275, 2334.

[16] Li, W., Church, R. L., \& Goodchild, M. F. (2014). The pcompact-regions problem. Geographical Analysis, 46(3), 250-273.

[17] Ligmann-Zielinska, A., Church, R. L., \& Jankowski, P. (2008). Spatial optimization as a generative technique for sustainable multiobjective land-use allocation. International Journal of Geographical Information Science, 22(6), 601-622.

[18] Lydersen, J. M., Collins, B. M., Brooks, M. L., Matchett, J. R., Shive, K. L., Povak, N. A., ... \& Smith, D. F. (2017). Evidence of fuels management and fire weather influencing fire severity in an extreme fire event. Ecological Applications, 27(7), 2013-2030.

[19] Moritz, M. A., Batllori, E., Bradstock, R. A., Gill, A. M., Handmer, J., Hessburg, P. F., ... \& Syphard, A. D. (2014). Learning to coexist with wildfire. Nature, 515(7525), 5866.

[20] Murray, A. T. (2021). Contemporary optimization application through geographic information systems. Omega, 99, 102176.

[21] Pais, C., Carrasco, J., Moudio, P. E., \& Shen, Z. J. M. (2021). Downstream protection value: Detecting critical zones for effective fuel-treatment under wildfire risk. Computers \& Operations Research, 131, 105252.

[22] Schoepfle, O. B., \& Church, R. L. (1991). A new network representation of a "classic" school districting problem. Socio-Economic Planning Sciences, 25(3), 189-197.

[23] Shirabe, T. (2005). A model of contiguity for spatial unit allocation. Geographical Analysis, 37(1), 2-16. 
[24] Stanislaus National Forest (2020). Social and Ecological Resilience Across the Landscape (SERAL). Briefing Paper, 2 pp.

[25] Stephens, S. L., Martin, R. E., \& Clinton, N. E. (2007). Prehistoric fire area and emissions from California's forests, woodlands, shrublands, and grasslands. Forest Ecology and Management, 251(3), 205-216.

[26] Tong, D., \& Murray, A. T. (2012). Spatial optimization in geography. Annals of the Association of American Geographers, 102(6), 1290-1309.
[27] Wright, J., ReVelle, C., \& Cohon, J. (1983). A multiobjective integer programming model for the land acquisition problem. Regional Science and Urban Economics, 13(1), 31-53.

[28] Wu, X., \& Murray, A. T. (2008). Spatial contiguity optimization in land acquisition. Journal of Land Use Science, 2(4), 243-25.
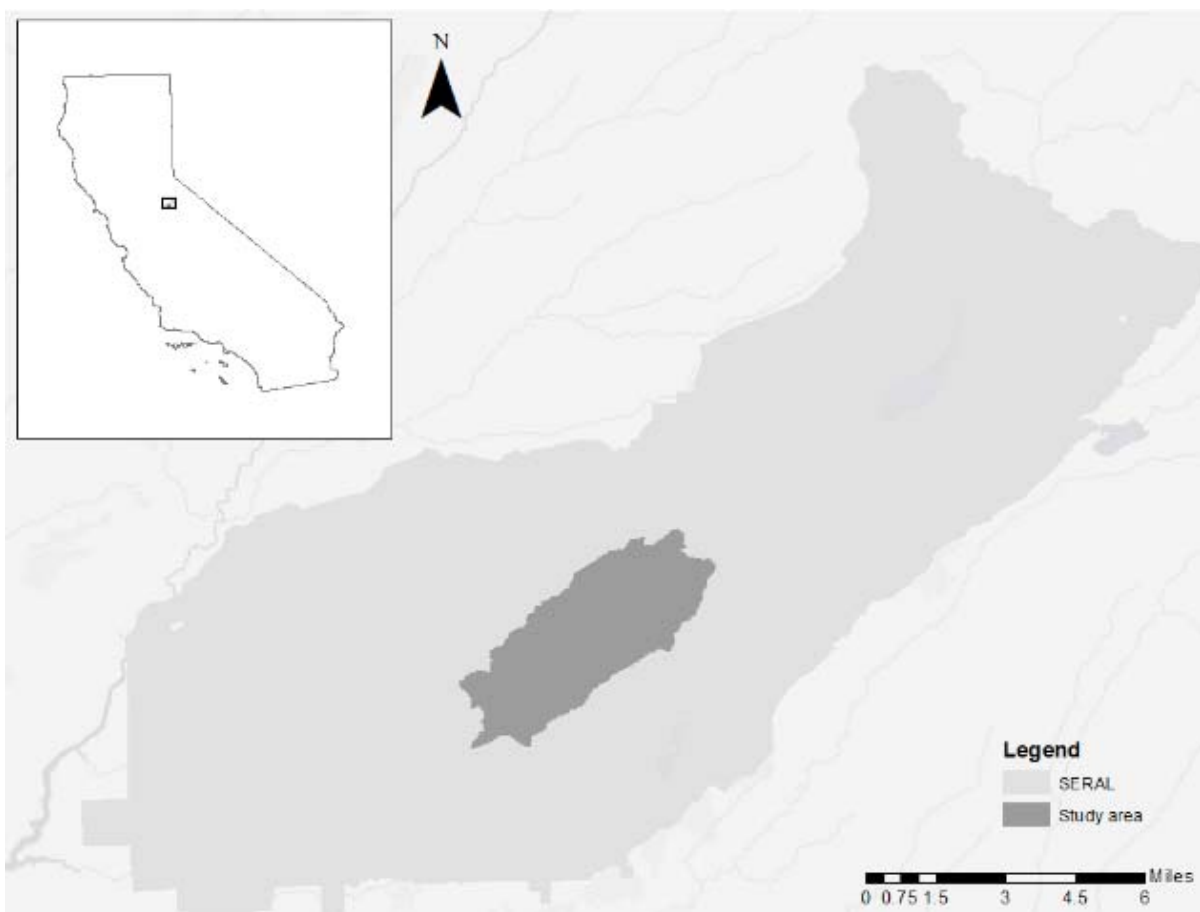

Figure 1: SERAL study region of the Stanislaus National Forest, with Potential Operational Delineations area used for analysis highlighted (Inset: location of region in California). 


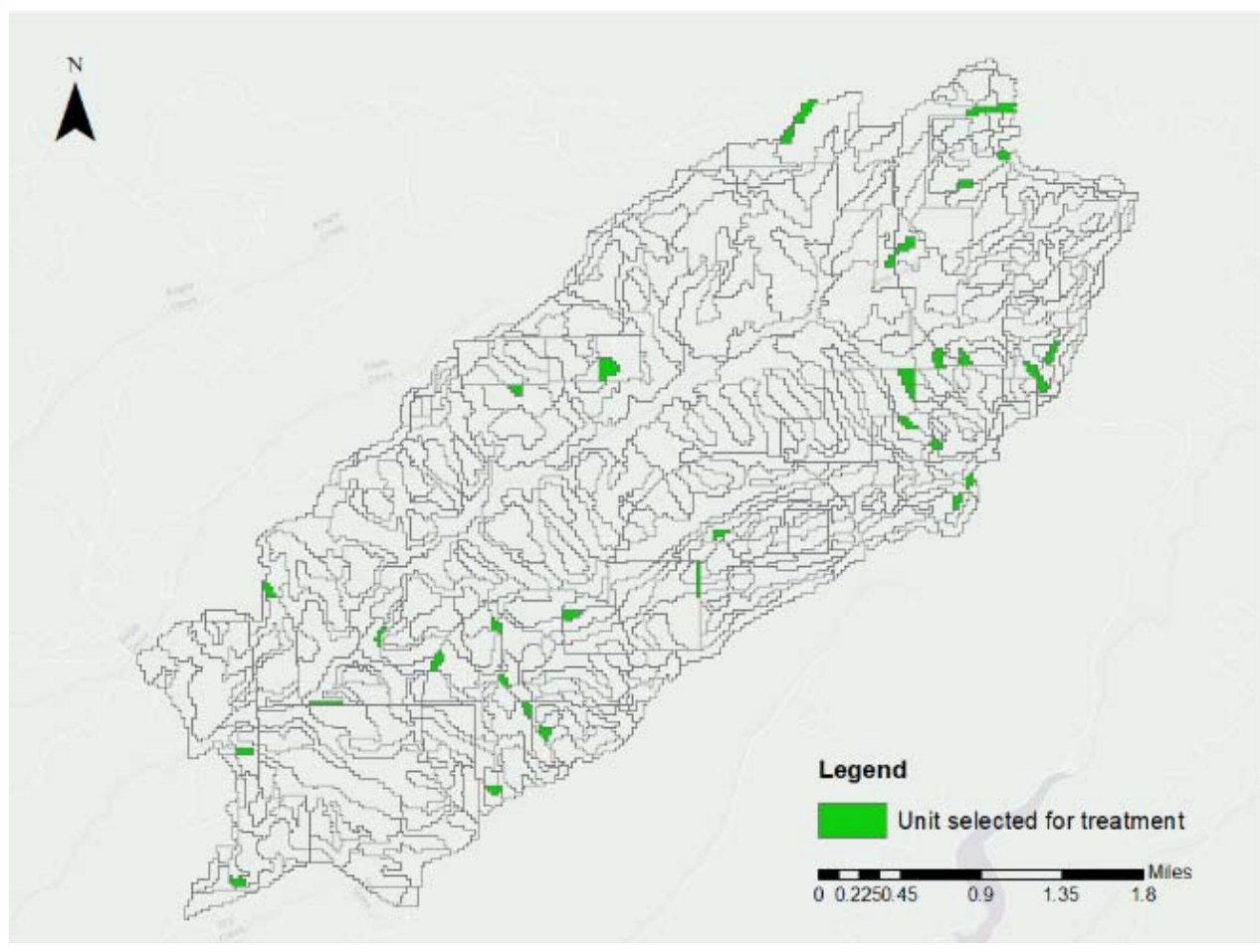

Figure 2: Land management units selected using the knapsack approach (without a contiguity requirement).

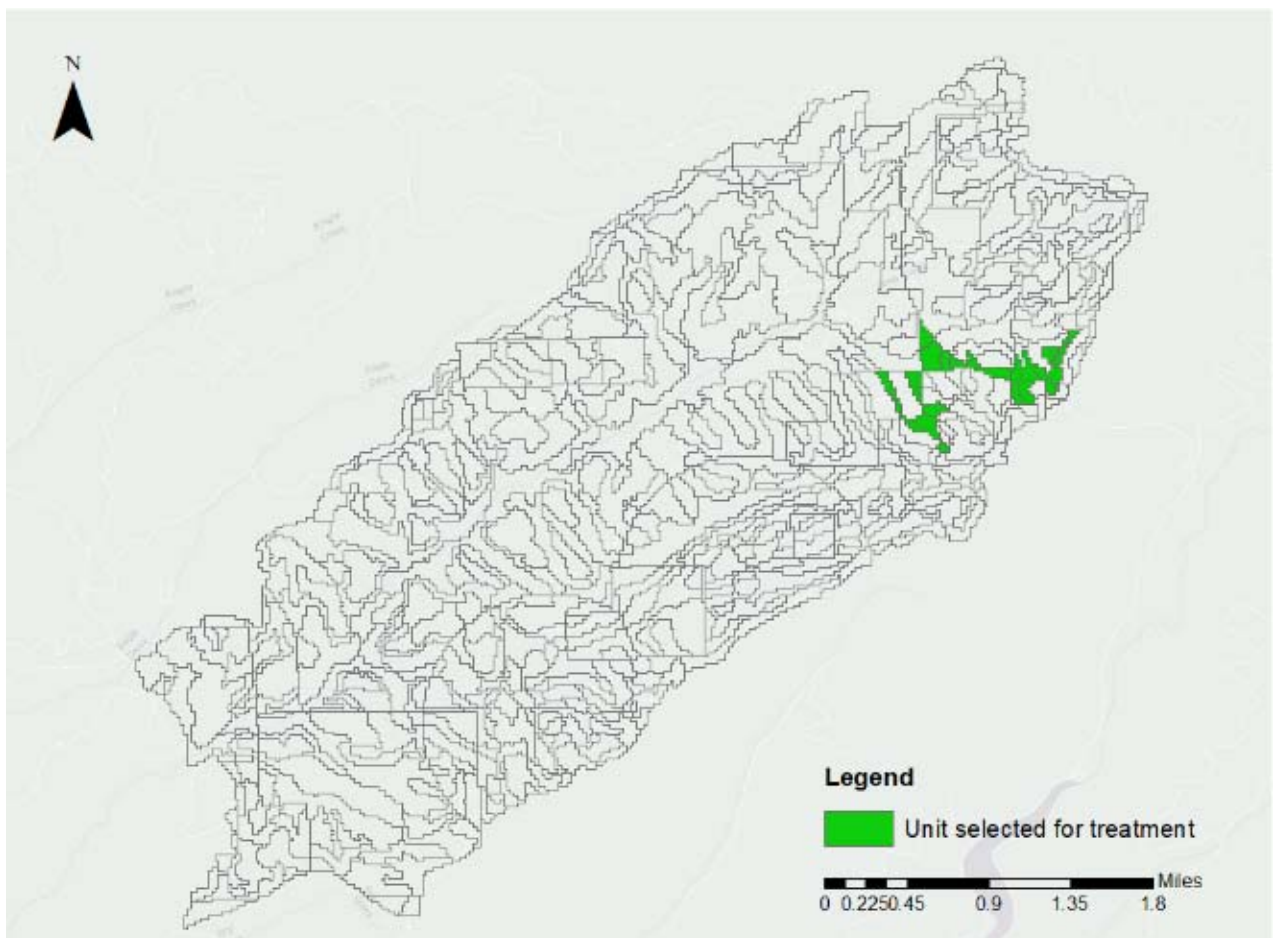

Figure 3: Landscape management units selected using the knapsack approach with a spatial contiguity requirement. 